\title{
Persian Vehicle License Plate Recognition Using Multiclass Adaboost
}

\author{
Mohammad Mahdi Dehshibi, Member, IACSIT, and Rahele Allahverdi
}

\begin{abstract}
Despite the success of License Plate Recognition (LPR) methods in the past decades, this problem is quite a challenge due to the diversity of plate formats and multiform outdoor illumination conditions during image acquisition. This paper presents a real-time and robust method for Persian license plate location and recognition. The proposed method consists of four main steps namely (I) Plate localization (II) Normalization, (III) Character segmentation, and (IV) Optical character recognition. First, all plates of the grabbed image are located rapidly and accurately using morphological operation and AdaBoost. After that, plates are normalized, and if they are skewed, then they will be aligned. In next step, convolution of each plate and a predefined binary mask is calculated, and then characters are segmented based on the obtained information. Finally, SAMME is utilized to classify extracted Persian numbers, alphabets, and words. In comparison with other methods, this system achieves high accuracy in plate localization, segmentation, and recognition. The success rate of the proposed method is $96.93 \%$ for plate localization utilizing morphological operation and AdaBoost, $\mathbf{9 8 . 7 5 \%}$ for character segmentation, and $94.5 \%$ for optical character recognition utilizing SAMME. Finally, the overall accuracy of the proposed method is examined $90.45 \%$.
\end{abstract}

Index Terms - Vehicle license plate recognition, persian OCR, multiclass adaBoost, character segmentation.

\section{INTRODUCTION}

The most important reason for the growth of research in the field of machine vision is producing applications that can be used for surveillance. One of these applications is automatic vehicle License Plate Recognition (LPR). This technology tends to be region-specific, owing to plate variation from place to place. Therefore, it is still considered as an open research field. LPR has a wide range of applications in surveillance and traffic systems since the license number is the primary, most widely accepted, human readable, mandatory identifier of motor vehicles. These applications include parking automation and parking security, traffic control management for recognizing vehicles that commit traffic violation and entering a restricted area without permission, occupying lanes reserved for public transport, crossing red light, breaking speed limits, etc. It can be also applied to automating fuel supplies in a filling station, motorway road tolling, and journey time measurement [1], [2], [4], [11].

In the proposed LPR system, as a vehicle approaches the

Manuscript received March 12, 2012; revised May 16, 2012.

M. M. Dehshibi is with the Faculty of Computer and IT, Islamic Azad University, Parand Branch, Tehran, Iran (e-mail: mohammad.dehshibi@piau.ac.ir).

R. Allahverdi is now with the Department of Computer, Islamic Azad University, Qazvin Branch, Qazvin, Iran (e-mail: r.allahverdi@qiau.ac.ir). camera takes a series of snapshots and stores them in a file. First, morphological operation is applied to the images to highlight the regions of interest. Then, AdaBoost is used to extract the plate accurately. Next, Median filter, which is a nonlinear operation, is applied to reduce noise and unwanted illumination effects. In the next step, convolution of a standard mask and plate is calculated in an iterative manner to extract all connected components of a plate. These components are Persian numbers and Persian alphabets. Note that for using alphabets, some constraints are defined in license plates, which we describe them in next section. Finally, a multi-class AdaBoost, called SAMME [15], is used to recognize the numbers and alphabet of plate. This system contends with a huge variety of lighting conditions, daytime, nighttime, sunlight, backlight, headlights, etc.

The rest of paper is organized as follows. In Section II, previous works related to the automatic LPR systems are reviewed. Section III describes how the proposed method extracts the plate from image, normalizes the size, orientation and the brightness of the plate and recognizes the optical characters. In Section IV, experimental results are described. Finally, this paper is concluded in Section V.

\section{PReVious Works}

Researchers have proposed several methods for three decades or so, which we briefly review them in this section. LPR systems can be mainly classified into three groups including edge-based, color-based, and texture-based algorithms. Popularity of the edge-based algorithms has increased among researchers due to their low computation complexity and simplicity. For instance, Duan et al. [6] counted edges on the image rows to find out regions of dense edges. Then, they applied a spatial analysis by morphology to the extracted region in order to connect the edges into LP regions. Bayoumi et al. [2] utilized edge density and background color to locate numbers, symbols and alphabets of an Egyptian plate. Their system depended on the characteristics of the number plate. Then, they applied projection functions to extract the license plate, and utilized a neural network in order to recognize the digits, symbols and alphabets.

Nonetheless, color-based algorithms, in addition to the edge-based algorithms, play an important role in license plates localization [5], [12], these methods depend more on the application conditions, and can be affected a lot by lighting conditions.

In [9], they used a SVM to train texture classifiers to detect image block that contains LP pixels. Kahraman et al. [8] applied Gabor filters to extract texture features in multi-scales and multi-orientations to describe the texture 
properties of LP regions. Sun et al. [13], proposed a new recognition method of vehicle license plates based on a Genetic Neural Network. As feature extraction is also important for improving the recognition rate of the network. Therefore, they used both structural and statistical features, which include mesh, direction line element and Zernike moments features. The main drawback of the texture-based algorithms is that they reduce their efficiency by multi-lingual factors.

\section{PROPOSED METHOD}

Two types of Persian license plate complicate the LPR systems. In all types a set, of characters and words including

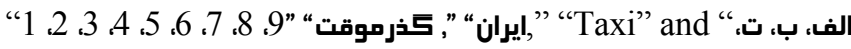

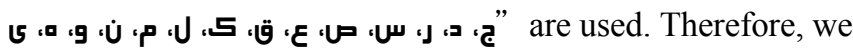
need several optical character recognition for identify numbers, letters and words in both languages of Persian and Latin. Fig. 1 illustrates samples of these two different types.

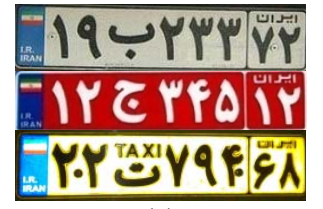

(a)

Fig. 1. (a) Formal license plates of Iranian's car (b) Temporary license plate

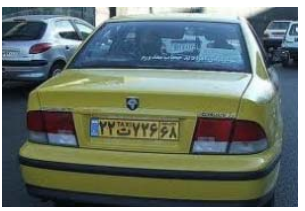

(a)

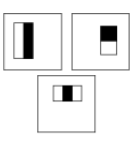

(b)

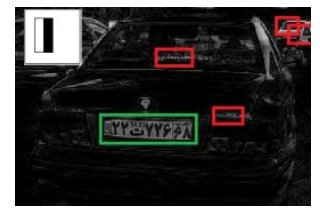

(c)
Fig. 2. (a) Captured image of a vehicle with crowded background (b) Haar like features (c) Result of applying morphological operators and plate extraction algorithms. Green box shows detected plate and red boxes show regions of interest which rejected by classifier.

Features of these types are as follows:

1. Type 1: this type contains Persian numbers, Persian letters, "ايران" word and Iran flag with different background color, i.e., white, yellow, and red. Note that public transport vehicles contain "Taxi" word, and their plates are yellow; see Fig. 1(a).

2. Type 2: this type contains Persian numbers and “"كذرموقت" word. This type uses for foreign vehicles which passing in Iran for six months; see Fig. 1(b).

The algorithm requires four primary steps for identifying a license plate. These steps are (I) Plate localization, which responsible for finding and isolating the plate on the picture. (II) Normalization in which the brightness and contrast of the images are adjusted. (III) Character segmentation, and (IV) Optical character recognition. These steps will be described in next sections.

\section{A. Plate Localization Using AdaBoost}

The plate localization plays an important role in the accuracy of the system. Many papers utilize edge information as well as morphological operation and some predefined constrained about plate size. However, in real world application these constraints may not occur. In this manner, we decide to utilize AdaBoost binary classifier with Haar like features which are demonstrated by Fig. 2(b). To extract the exact location of plate, first, erode operation is applied to the original image with a rectangle morphological structure. Then, the eroded image is dilated by the same structure, and the result is subtracted from the original image.

Fig. 2(c) shows the result of applying morphological operators to the original image. As is evident, the result contains more regions than the interest region. To increase the accuracy of the proposed method an AdaBoost binary classifier with Haar like features [7] is utilized. The value of a two-rectangle feature is the difference between the sums of the pixels within two rectangular regions. A three-rectangle feature computes the sum within two outside rectangles subtracted from the sum in a center rectangle. In order to increase the speed of calculating features, integral image of the original images is computed. Integral image is used by Viola and Jones [14] for the first time.

The proposed classifier is a ten-layer cascade of AdaBoost classifiers which included a total of 1800 features. The first classifier in the cascade is constructed such that 5 features are used and rejects about $80 \%$ of non-plates while correctly detecting close to $100 \%$ of plates. The next classifier has 27 features and rejects $90 \%$ of non-plates while detecting almost $100 \%$ of plates. The next eight layers are 221 -feature classifiers. As it shown in Fig. 2 (c), to detect the plate, the image is scanned by a sub-window containing a Haar-like feature. Based on each Haar-like feature, a weak classifier is defined which finally makes the strong classifier.

\section{B. Plate Normalization and Character Segmentation}

The first step in plate normalization is to detect whether the region of interest (ROI) is skewed. To achieve this, the centre point of image is calculated (1).

$$
\mathrm{C}_{\mathrm{x}}=R O I_{\text {width }} / 2, \mathrm{C}_{\mathrm{y}}=R O I_{\text {height }} / 2
$$

Then, two vectors from the right and two vectors from the left vertices of the plate's corner and extracted region's corner are created, respectively (Fig. 3(b)). Finally, the left and right angle, $\theta_{\text {left }}$ and $\theta_{\text {right }}$, are calculated, and the ROI is rotated on the greater angle (2). Fig. 3(c) shows the aligned plate.

$$
\begin{array}{r}
\theta_{\text {left }}=\arccos \left(v_{\text {left }}^{1} \cdot v_{\text {left }}^{2} /\left\|v_{\text {left }}^{1}\right\|_{2} \times\left\|v_{\text {left }}^{2}\right\|_{2}\right) \times \frac{180}{\pi} \\
\theta_{\text {right }}=\arccos \left(v_{\text {right }}^{1} \cdot v_{\text {right }}^{2} /\left\|v_{\text {right }}^{1}\right\|_{2} \times\left\|v_{\text {right }}^{2}\right\|_{2}\right) \times \frac{180}{\pi} \\
\theta=\max \left\{\theta_{\text {left }}, \theta_{\text {right }}\right\} \\
{\left[\begin{array}{c}
x^{\prime} \\
y^{\prime}
\end{array}\right]=\left[\begin{array}{cc}
\cos \theta & -\sin \theta \\
\sin \theta & \cos \theta
\end{array}\right] \times\left[\begin{array}{l}
x \\
y
\end{array}\right]}
\end{array}
$$

where $\theta$ is rotation angle, $\mathrm{x}$ and $\mathrm{y}$ are coordinates of the original image, $x$ ' and $y$ ' are coordinates of the transformed image, $v^{1}$ is the vector which lies across the centroid to vertices of the plate's corner, and $v^{2}$ is the vector which lies 
across the centroid to vertices of the region's corner, $|$.$| is the$ inner product of two vectors and $\|.\|_{2}$ is the norm of vector.

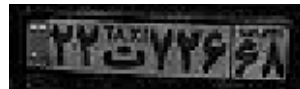

(a)

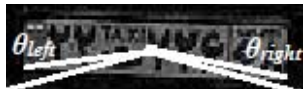

(b)

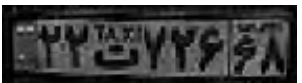

(c)

Fig. 3. (a) Region of interest (b) The left and right vectors which help to detect whether the plate is skewed (c) Aligned plate. In this sample, the plate is rotated by 2 degrees in a counter-clockwise direction.

The final stage in normalization is decreasing the system sensitivity to the noise, illumination variations and image scale. To achieve this, Median filter is applied to the aligned plate. Median filter is a nonlinear operation that is more effective than convolution when the goal is to simultaneously reduce noise and preserve edges. After filtering the ROI, this region is scaled to 300 by 80 pixels.

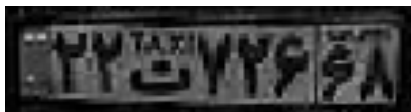

(a)

1

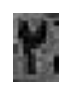

(c)

Fig. 4. (a) A Sample plate (b) A 8-connected mask (c) Segmented characters which are extracted by Connected Component Labeling Algorithm.

\section{SAMME Algorithm}

1. Initialize the observation weights $\mathrm{w}_{\mathrm{i}}=1 / \mathrm{n}, \mathrm{i}=1, \ldots, \mathrm{n}$.

2. For $\mathrm{m}=1$ to $\mathrm{M}$ :

(a) Fit a classifier $T^{(m)}(x)$ to the training data using weights $w_{i}$

(b) Compute

$$
\operatorname{err}^{(m)}=\sum_{i=1}^{n} w_{i} \mathrm{II}\left(c_{i} \neq T^{(m)}\left(x_{i}\right)\right) / \sum_{i=1}^{n} w_{i}
$$

(c) Compute

$$
\alpha^{(m)}=\log \frac{1-e r r^{(m)}}{e r r^{(m)}}+\log (K-1)
$$

(d) Set

$w_{i} \leftarrow w_{i} \cdot \exp \left(\alpha_{(m)} \cdot \operatorname{II}\left(\mathrm{c}_{\mathrm{i}} \neq \mathrm{T}^{(\mathrm{m})}\left(\mathrm{x}_{\mathrm{i}}\right)\right)\right), i=1, \ldots, n$.

(e) Renormalize $\mathrm{w}_{\mathrm{i}}$

3. Output

$$
C(x)=\arg \max _{k} \sum_{m=1}^{M} \alpha^{(m)} \mathrm{II}\left(T^{(m)}(x)=k\right)
$$

In character segmentation, first, we need to obtain the precise bounding boxing of each character before recognition (as shown in Fig. 4(c)). In this process, a binary 5 by 5 pixel 8-connected mask is defined (as shown in Fig. 4(b)), and convolution of plate and the mask is calculated in order to reduce the number of connected components by smoothing the image. All pixels of a plate's character share similar pixel intensity values, and are in some way connected with each other.

The mask moves along a row until it comes to a point for which its value equals to range of plates character. When this is true, it examines the eight neighbors of this pixel that have already been encountered in the scan. Based on this information, the labeling occurs.

\section{Plate Character Recognition}

Recognizing Farsi digits, alphabet, and three words including "ايران," “"كذرموقت "Taxi” are more difficult and complicated than Latin one. This step is the last stage in the LPR system where characters and words are recognized automatically using Multiclass AdaBoost approach, called SAMME. The input feature of the machine is obtained from pixels of any segmented character. In order to simplify the process of input features, each segmented character is scaled to a fix size of 30 by 80 pixels by padding with white color. SAMME proceeds as follows:

SAMME is very similar to AdaBoost with a major difference in stage (c). For $\alpha(\mathrm{m})$ to be positive, we only need $\left(1-\right.$ err $\left.^{(\mathrm{m})}\right)>1 / K$, where $\mathrm{K}$ is the number of classes.

Consequently, the new algorithm puts more weight on the misclassified data points in (2d) than AdaBoost, and the new algorithm also combines weak classifiers a little differently from AdaBoost, i.e. differ by $\log (K-1) \sum_{m=1}^{M} \mathrm{II}\left(T^{(m)}(x)=k\right)$. The term $\log (\mathrm{K}-1)$ in $(2 \mathrm{c})$ makes the new algorithm equivalent to fitting a forward stage-wise additive model using a multi-class exponential loss function. In our application, the classifier is 30 classes classifier which has an input vector with size of 2400 .

\section{EXPERIMENTS}

In order to illustrate the efficiency of the proposed method a set of experiments is conducted. These experiments involve all sub algorithms which support the final goal, i.e., Plate Localization, Plate Normalization and Character Segmentation, and OCR. The overall accuracy of the proposed method is calculated as follows (3):

$$
\begin{gathered}
\text { accuracy } \%=(\text { Localization }) \times(\text { Segmentation }) \times \\
(\text { Recognition }) \\
(\text { number of plate's component })
\end{gathered}
$$

As it is mentioned in Section 3.1, locating the plate position is done utilizing AdaBoost. In learning process, 1176 image plates of Type 1, 62 image plates of Type 2 and 4500 non-plate images are used. The plate localization sub-algorithm, which it is responsible for finding the plate on the picture, has $96.93 \%$ accuracy. In order to expect reasonable results from a plate localization algorithm, the processed images should contain a plate with reasonable good spatial resolution, sharpness, lighting conditions, and capturing position. Shortcoming of these items can highly affect the localization process as in our experiments.

Segmentation algorithm in plates of Type 2 is more difficult than Type 1. Because in Type 2, we have two

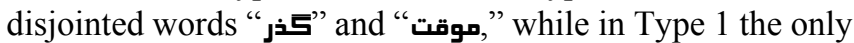
disjointed word is "ايرانl," and even "الف" is inscribed as on the plate. The accuracy of this sub algorithm is 98.75 percent. Components of 1185 plates are correctly segmented. The highest failure rate relates to plates of Type 2 where just 52 plates among 60 plates are correctly segmented.

To demonstrate the efficiency of recognition phase, the proposed method is compared with two popular classifiers, 
i.e., ANN [3] and AdaBoost.MH [10]. The AdaBoost.MH algorithm converts our 30-class problem into that of estimating a two-class classifier on a training set 30 times as large, with an additional feature defined by the set of class labels. Hence, the computational cost is 30 times larger than the computational cost of SAMME.

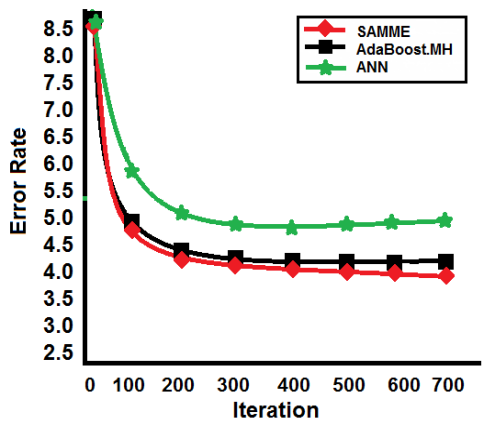

Fig. 5. Test errors for SAMME, adaboost.MH and artificial neural network on car dataset.

Fig. 5 compares SAMME, AdaBoost.MH, and ANN for recognition of one digit. The standard error is approximated by $\sqrt{\text { test }_{\text {error }} \cdot\left(1-\text { test }_{\text {error }}\right) / \text { size }_{\text {test }}}$, where is the test error, and is the size of testing data. As is shown in Fig. 5, the best recognition rate of the proposed method for one of components is $96 \%$. When this experiment is performed on other segmented component, the average recognition is $94.5 \%$. Therefore, the overall accuracy of the proposed method based on (3) is $90.45 \%$.

\section{CONCLUSION}

The most important reason for the growth of research in the field of machine vision is producing applications that can be used for surveillance. One of these applications is automatic vehicle License Plate Recognition (LPR). Despite the success of License Plate Recognition (LPR) methods in the past decades, this problem is quite a challenge due to the diversity of plate formats and multiform outdoor illumination conditions during image acquisition. In this paper we present a real-time and robust method for Persian license plate location and recognition. The proposed method consists of four main steps namely (I) Plate localization (II) Normalization, (III) Character segmentation, and (IV) Optical character recognition. In order to illustrate the efficiency of the proposed method, we conduct a set of experiments. The success rate of the proposed method is $96.93 \%$ for plate localization utilizing morphological operation and AdaBoost, $98.75 \%$ for character segmentation, and $94.5 \%$ for optical character recognition by utilizing SAMME. Finally, we obtained an overall accuracy of 90.45\% in Persian vehicle license plate recognition.

\section{REFERENCES}

[1] C. N. E. Anagnostopoulos, I. E. Anagnostopoulos, V. Loumos, and E. Kayafas, "A license plate-recognition algorithm for intelligent transportation system applications," in IEEE Trans. Intell. Transp. Syst, vol. 7, pp. 377-391, 2006.

[2] S. Bayoumi, E. Korany, and S. Fathy, "License Plate Recognition system for Egyptian Car Plates," in proc. of IVPCV-10, pp. 139-146, 2010.

[3] A. Broumandnia and M. Fathy, "Application of pattern recognition for Farsi license plate recognition," in ICGST-GVIP Journal, vol. 5, pp. 25-31, 2005.

[4] P. Davies, N. Emmott, and N. Ayland, "License plate recognition technology for toll violation enforcement," in Inst. Elect. Eng. Colloquium Image Analysis for Transport Applications, pp. 1-5, 1990.

[5] K. Deb, H. Lim, S. J. Kang, and K. H. Jo, "An Efficient Method of Vehicle License Plate Detection Based on HSI Color Model and Histogram," in LNCS, vol. 5579, pp. 66-75, 2009.

[6] T. D. Duan, T. L. H. Du, T. V. Phuoc, and N. V. Hoang, "Building an automatic vehicle license plate recognition system," in Proc. of Int. Conf. on Computer Science RIVF, pp.59-63, 2005.

[7] Y. Freund and R. Schapire, "A decision theoretic generalization of on-line learning and an application to boosting," in Journal of Computer and System Sciences, vol. 55, no. 1, pp. 119-139, 1997.

[8] F. Kahraman, B. Kurt, and M. G. Okmen, "License plate character segmentation based on the Gabor transform and vector quantization," in $L N C S$, vol. 2869, pp.381-388, 2003.

[9] K. I. Kim, K. Jung, and J. H .Kim, "Color texture-based object detection: an application to license plate localization," in LNCS, vol. 2388, pp.293-309, 2002.

[10] R. Schapire and Y. Singer, "Improved boosting algorithms using confidence-rated prediction," in Machine Learning, vol. 37, no. 3, pp. 297-336, 1999.

[11] T. Sirithinaphong and K. Chamnongthai, "The recognition of car license plate for automatic parking system," in Proc. 5th Int. Symp. Signal Processing and its Applications, pp. 455-457, 1998.

[12] X. Shi, W. Zhao, and Y. Shen, "Automatic license plate recognition system based on color image processing," in LNCS, vol. 3483, pp.1159-1168, 2005.

[13] G. Sun, C. Zhang, W. Zou, and G. Yu, "A new recognition method of vehicle license plate based on Genetic Neural Network," in Proc. of 5th IEEE ICIEA, pp.1662 - 1666, 2010.

[14] P. Viola and M. Joens, "Rapid object detection uses a boosted cascade of simple features," in IEEE CVPR, pp. 511-518, 2001.

[15] J. Zhu, H. Zou, S. Rosset, and T. Hastie, "Multi-class AdaBoost," Statistics and Its Interface, vol. 2, pp. 349-360, 2009.

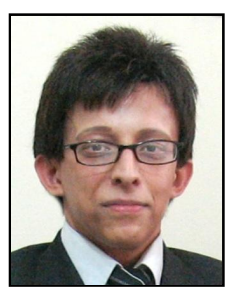

Mohammad Mahdi Dehshibi received the Master degree in computer engineering, from Islamic Azad University, Qazvin Branch in 2010, and he is a Ph.D candidate at Islamic Azad University, Science and Research Branch. While pursuing the Master degree, he was a Research Assistant with the IRIB University, in charge of several master projects in image analysis, pattern recognition, and medical image processing. His research interests are in facial and medical image processing, multimedia data processing, pattern recognition, and computer vision. Mohammad Mahdi Dehshibi is an associated member of IEEE Computer Society, an Associate member of DISWC.

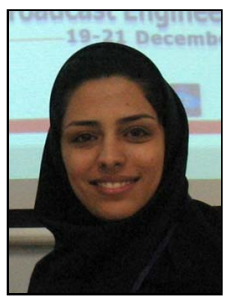

Rahele Allahverdi received the B.S. degree in computer engineering, from Islamic Azad University, Karaj Branch in 2008. She is a M.S. student in Islamic Azad University, Qazvin Branch. Her research interests include pattern recognition, machine vision and image processing. 